\title{
The Dynamic Characteristic of Printed Circuit Board with the Use of the Concept of Simplified Representative Volume Elements*
}

\author{
Taek Yul $\mathrm{OH}^{* *}$, Hyun Suk SEO ${ }^{* * *}$, Young-Ha KWON** and Yoon Hyuk KIM**
}

\begin{abstract}
PCB (Printed Circuit Board)s are designed in various sizes and shapes, use variety of processes and materials, and perform a variety of electrical, structural, and some times thermal functions. The major elements of PCBs are the fabric, the resin, and the metal foil (usually copper). The auxiliary elements are the adhesion promoters or treatments that are applied to the fabric and to the copper to assure maximum adhesion of the resin to the fabric and to the copper. Each copper layer has complicated and different pattern to correctly operate for its mission. In that case, the stiffness of PCBs are affected by the copper layers. By reasoning of this complicated copper layer pattern, it is difficult to determine the PCB stiffness. SAR (Solar Array Regulator) for Korea Leo Earth Observation \& Science Satellites Program uses two PCBs of different types and sizes. These PCBs are composed of the resin system and copper layers, and not used the fabric. For this study, arm converter board applied to the SAR components is considered. In this study, the methodology of calculation of the PCB stiffness for SAR component is suggested considering the concept of simplified representative volume element and this property will be correlated with the vibration test results.
\end{abstract}

Key Words: PCB (Printed Circuit Board), Random Vibration, RVE (Representative Volume Element), Solar Array Regulator

\section{Introduction}

Provision of electric power for space vehicles is, perhaps, the most fundamental requirement for the satellite payload. Power-system failure necessarily results in the loss of a space mission, and it is interesting to note that many of the early satellite systems failed due to such loss.

Especially, SAR (Solar Array Regulator) is an important component of EPS (Electric Power Subsystem) to convert the solar array voltage to main bus voltage.

Such electronic components should be designed considering the launching environments like a random vibration, acoustic, air pressure and pyro-shock. During the launching, random vibration is a key parameter to confirm the reliability of structures such as SAR electronic com-

* Received 1st December, 2004 (No. 04-5195)

** College of Advanced Technology, Kyung-Hee University, Kyunggi 449-701, Korea. E-mail: tyoh@khu.ac.kr; yhkwon@khu.ac.kr; yoonhkim@khu.ac.kr

*** Space Development \& Research Center, Korea Aerospace Industries, \#48-6, Moonpyung-dong, Daeduk-ku, Daejon 306-220, Korea. E-mail: shs0315@freechal.com ponents.

This random vibration can yield some board deflections, so that this deflection may cause the failure of solder joint $^{(1)}$. In order to prevent such failure of solder joint, the random vibration environment has to be controlled ${ }^{(1)}$.

Electronic equipment may be subjected to many different forms of vibration during service life and in transportation and handling, which may include many different types of failures, as described by Steinberg ${ }^{(1)}$. Roberts and Stillo $^{(2)}$ used finite element modeling to analyze the vibration fatigue of ceramic capacitor's leads under random vibration. Barker et al. ${ }^{(3),(4)}$, Sidharth and Barker proposed some analytical methods to estimate the vibration fatigue life of leaded surface mount components. Ham and Lee ${ }^{(5)}$ developed a fatigue-testing system to study the integrity of electronic packaging subjected to mechanical vibration. In order to develop reliability assessment and design-for-reliability methodologies against vibration fatigue, research work was carried out for a few years. But these studies focused on the parts and components level under a certain vibration and thermal load. The estimation of exact model for PCBs was not included. 
Environmental compatibility analyses such as the thermal and structural analysis are often conducted to verify hardware design compliance with mission environments. Also, to accurately simulate the environmental sequence, the hardware testing such as thermal vacuum, vibration testing should be performed. Understanding the PCB structures is an important factor of mechanical design. Normally, PCBs (Printed Circuit Boards) are composed of multi-layered copper $(\mathrm{Cu})$ plates and glass epoxy materials ${ }^{(11)}$. Since the copper layers and patterns are directly related to the board dynamic characteristics, an alternate way for the determination of PCB mechanical properties considering the complexity of this patterns and layers will be needed. However, to our knowledge, the stiffness calculation of PCB multi-layers in order to estimate the maximum deflection under random vibration load has not been studied yet.

In this study, the Copper $(\mathrm{Cu})$ layer regarded as a simplified volume element, is introduced and the representative stiffness of printed circuit board is obtained using the classical lamination theory $(\mathrm{CLT})^{(12),(13)}$. Also we construct the mathematical model using this composite mechanics and correlate the model between random vibration test and analysis results.

This paper describes the theoretical consideration of mechanical performance of printed circuit board and the validity of theoretical equations comparing with the random vibration test results. And it can be stated that the suggested methodology will be used in the numerical model of PCB under random forces.

\section{Structure Analysis of Printed Circuit Board}

\subsection{The structural characteristic of printed circuit board}

Printed circuit boards, commonly called PCBs, are sometimes referred to as the baseline in electronic packaging. It serves a wide variety of functions. An organic rigid PCBs laminate consists of three major elements and some auxiliary elements. The major elements are the fabric, the resin and the metal foil (usually copper). The auxiliary elements are the promoters or treatments that are applied to the fabric and to the copper to assure maximum adhesion of the resin to the fabric and to the copper. Normally, glass epoxy is two different type of material; one is glass fiber reinforced plastic and the other is resin only, for examples, epoxy, FR-4, polyimide and etc.

Three type of SAR models are developed: DM (Development Model), PM (Proto-type Model) and FM (Flight Model). For this study, PCBs is used as the traditional rigid PCBs. The glass epoxy is FR-4 resin only, and foil is copper material. The following figures show the pattern of PCB copper layers as SAR PM.

The number of copper layers and patterns of $\mathrm{PCB}$ for PM and FM are nearly the same for each model. But,

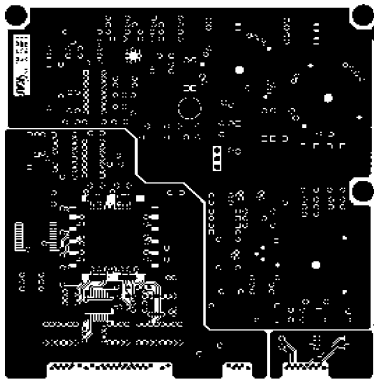

(a) The 1st copper layer

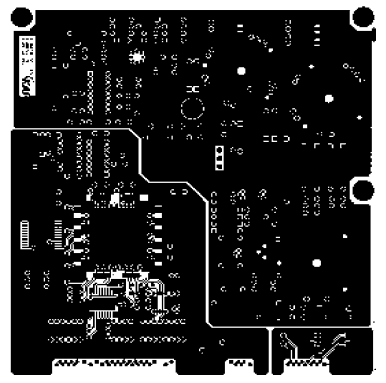

(b) The 10th copper layer
Fig. 1 Outer $\mathrm{Cu}$ layer pattern of 1 st and 10th layer

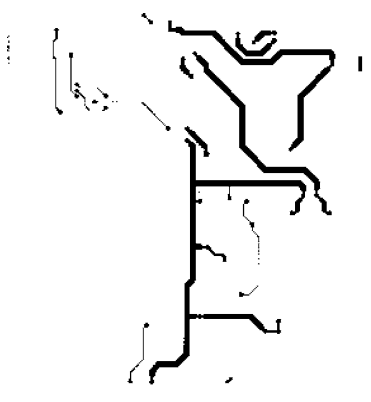

(a) The 3rd copper layer

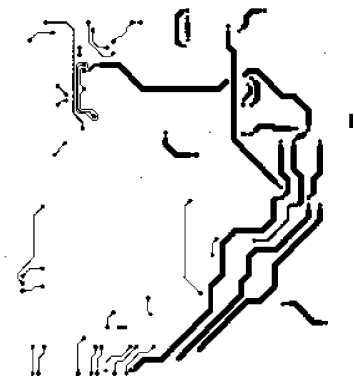

(b) The 7th copper layer
Fig. 2 Inner $\mathrm{Cu}$ layer pattern of $3 \mathrm{rd}$ and 7 th layer

DM PCB has 8 copper layers. The middle layers of 2 to 7 for DM and 2 to 9 for PM \& FM is of 34 micro meter thickness and each of outer two layer (1 and 8 layer for DM \& 1 and 10 layer for PM and FM) is of 68 micro meter thickness. Total thickness of each PCB is 0.068 in $(1.7272 \mathrm{~mm})$ for 8 layers and $0.090 \mathrm{in}(2.286 \mathrm{~mm})$ for 10 layers.

\subsection{F.E. modeling of PCB using composite lamina- tion theory}

To verify the structural reliability of the SAR component for satellites, analytical approaches are widely used. For this study, F.E. Method is suggested. However, the complexity of copper layer pattern provides inevitable error in making a numerical model of PCBs. To reduce the uncertainty of structural model for PCBs, some appropriate method is needed. This chapter shows the method to describe more accurate model for PCBs using micromechanics and classical composite theory.

As shown in Fig. 3, the elementary mechanics of composite materials for $E_{1}$ and $v_{12}$ are good enough for design use. The corresponding models for $E_{2}$ and $G_{12}$ are of questionable value, however, because they are based on invalid assumptions and agreement with experimental results is generally poor. We will now discuss several refinements of the elementary mechanics of materials models.

Due to the simplified RVE (Representative Volume Elements) that was used for the elementary mechanics of materials approach, the resulting equations were not tied to any particular fiber-packing geometry. Since the results 


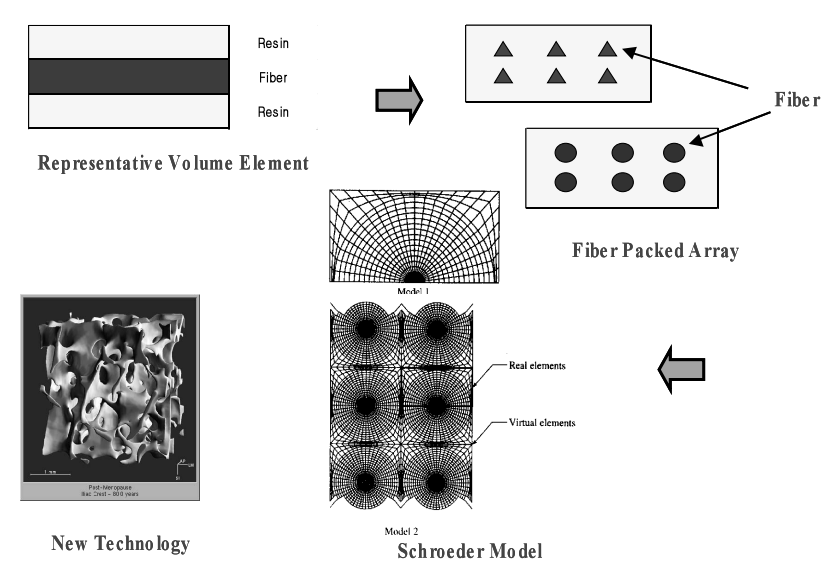

Fig. 3 History of micro mechanics for composite materials

for $E_{1}$ and $v_{12}$ were so favorable, we can conclude that those properties must be essentially independent of fiberpacking geometry. By the same reasoning, it appears that $E_{2}$ and $G_{12}$ may be more sensitive to fiber-packing geometry. Thus, the assumption of a specific fiber-packing array is one possible refinement of the models. Normally, real composite has the random-packing arrays and volume fraction. Modern scientists developed a refined model for transverse and shear properties based on a square fiberpacking array and a method of dividing the RVE into subregions.

For this study, elementary mechanics of micro-scope composite material will be adopted using simplified RVE.

According to the micro mechanic of composite material, we suggest that copper layer is considered as representative volume elements. So for the copper layer regarded as a simplified volume elements suggested, is the following single lamina theory of the micro mechanics for composite material. But in this study, more refined fiber packing array model is not needed considering the simple structural characteristics of copper layers. As expected, the copper material is highly isotropic, with $E_{1}=E_{2}$. So the copper layer is regarded as a simplified volume elements using RVE method.

The above figure shows the schematics of SAR PCB considering the complicated layer patterns as simplified volume. Each of copper layer pattern and area density was written by $\mathrm{CAD}$ program for the $\mathrm{Cu}$ pattern design, for examples, E-CAD, OrCAD, AutoCAD or etc. The method for the equivalent stiffness calculation of $\mathrm{PCB}$ can be easily obtained using the area density of $\mathrm{Cu}$ layer for the nominal size of PCBs. If $\mathrm{Cu}$ layer has $50 \%$ area density with 34 micro meter, then the $\mathrm{Cu}$ layer can be 17 micro meter for the nominal size of PCBs. That means that we obtained the ratio of section area to nominal board size. And this ratio could be transferred from the complicated copper pattern to the average copper thickness. The representative stiffness of printed circuit board was obtained using 'classical lamination theory' for composite materi- als considering the above assumption.

According to the 'CLT', tangential displacements $u$ and $v$ are linear functions of the $z$ coordinate. So the displacement can be expressed as

$$
\begin{aligned}
& u=u^{0}(x, y)+z F_{1}(x, y) \\
& v=v^{0}(x, y)+z F_{2}(x, y) \\
& w=w^{0}(x, y)=w(x, y)
\end{aligned}
$$

By substituting Eq. (1) into the strain-displacement equations for the transverse shear strains, we find that

$$
\begin{aligned}
& \gamma_{x z}=\frac{\partial u}{\partial z}+\frac{\partial w}{\partial x}=F_{1}(x, y)+\frac{\partial w}{\partial x}=0 \\
& \gamma_{y z}=\frac{\partial v}{\partial z}+\frac{\partial w}{\partial y}=F_{2}(x, y)+\frac{\partial w}{\partial y}=0
\end{aligned}
$$

and

$$
F_{1}=-\frac{\partial w}{\partial x} \quad F_{2}=-\frac{\partial w}{\partial y}
$$

By substituting Eqs. (1) and (3) into the straindisplacement relations for the in-plane strains, we find that

$$
\begin{aligned}
& \varepsilon_{x}=\frac{\partial u}{\partial x}=\varepsilon_{x}^{0}+z \kappa_{x} \\
& \varepsilon_{y}=\frac{\partial v}{\partial y}=\varepsilon_{y}^{0}+z \kappa_{y} \\
& \gamma_{x y}=\frac{\partial u}{\partial y}+\frac{\partial v}{\partial x}=\gamma_{x y}^{0}+z \kappa_{x y}
\end{aligned}
$$

where the strains on the middle surface are

$$
\varepsilon_{x}^{0}=\frac{\partial u^{0}}{\partial x} \quad \varepsilon_{y}^{0}=\frac{\partial v^{0}}{\partial y} \quad \gamma_{x y}^{0}=\frac{\partial u^{0}}{\partial y}+\frac{\partial v^{0}}{\partial x}
$$

and the curvatures of the middle surface are

$$
\kappa_{x}=-\frac{\partial^{2} w}{\partial x^{2}} \quad \kappa_{y}=-\frac{\partial^{2} w}{\partial y^{2}} \quad \kappa_{x y}=-2 \frac{\partial^{2} w}{\partial x \partial y}
$$

$\kappa_{x}$ is a bending curvature associated with bending of the middle surface in the $x z$ plane and $\kappa_{y}$ is a bending curvature associated with bending of the middle surface in the $y z$ plane. $\kappa_{x y}$ is a twisting curvature associated with outof-plane twisting of the middle surface, which lies in the $x y$ plane before deformation.

Since Eq. (4) give the strains at any distance $z$ from the middle surface, the stresses along arbitrary $x y$ axes in the $k$ th lamina of a laminate may be found by substituting Eq. (4) into the lamina stress-strain relationships as follows :

$$
\left\{\begin{array}{l}
\sigma_{x} \\
\sigma_{y} \\
\tau_{x y}
\end{array}\right\}=\left[\begin{array}{lll}
\bar{Q}_{11} & \bar{Q}_{12} & \bar{Q}_{16} \\
\bar{Q}_{12} & \bar{Q}_{22} & \bar{Q}_{26} \\
\bar{Q}_{16} & \bar{Q}_{26} & \bar{Q}_{66}
\end{array}\right]_{k}=\left\{\begin{array}{c}
\varepsilon_{x}^{\mathrm{o}}+z \kappa_{x} \\
\varepsilon_{y}^{\mathrm{o}}+z \kappa_{y} \\
\gamma_{x y}^{\mathrm{o}}+z \kappa_{x y}
\end{array}\right\}
$$

where the subscript $k$ refers to the $k$ th lamina. Comparing the laminated plate stresses in Eq. (7) with the laminated beam stress, we notice several differences. The laminated beam analysis only gives the uniaxial stress due to the bending curvature, whereas the laminated plate analysis gives the two-dimensional lamina stress due to bending 


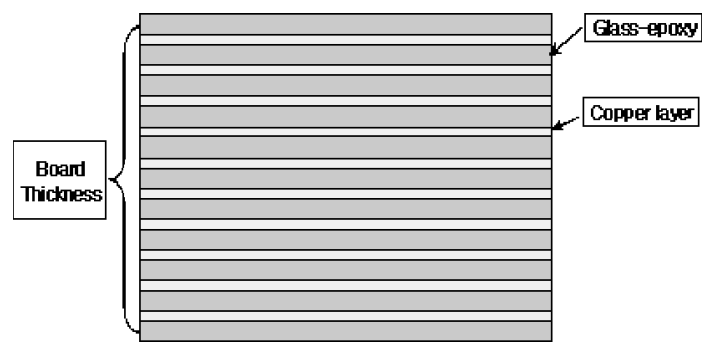

Fig. 4 Schematic of PCB layer

and twisting curvatures and to the midplane biaxial extension and shear. In addition, the laminated plate analysis includes the stresses due to shear coupling.

In the laminated beam analysis for lamina stress is seen to be of limited practical use because the curvature is not generally known and is difficult to measure. Thus, the lamina stress was related to the applied bending moment by using the static equilibrium relationship. In the laminated plate analysis the midplane strains and curvatures in the Eq. (7) must also be related to applied forces and moments by static equilibrium equations in order to make these equiations more useful.

But in the laminated plate analysis, it is convenient to use forces and moments per unit length rather than forces and moments themselves.

For example, the force per unit length, $N_{x}$, is given by

$$
N_{X}=\int_{-t / 2}^{t / 2} \sigma_{X} d z=\sum_{k=1}^{N}\left\{\int_{Z_{k-1}}^{Z_{k}}\left(\sigma_{x}\right)_{k} d z\right\}
$$

and the moment per unit length, $M_{x}$, is given by

$$
M_{x}=\int_{-t / 2}^{t / 2} \sigma_{x} z d z=\sum_{k=1}^{N}\left\{\int_{Z_{k-1}}^{Z_{k}}\left(\sigma_{x}\right)_{k} z d z\right\}
$$

where $t$ : laminate thickness

$\left(\sigma_{x}\right)_{k}$ : stress in the $k$ th lamina

$Z_{k-1}$ : distance from the middle surface to inner surface of the $k$ th lamina

$Z_{k}$ : corresponding distance from the middle surface to outer surface of the $k$ th lamina, as shown in Fig. 4

Substituting the lamina stress-strain relationships from Eq. (7) to Eqs. (8) and (9), respectively, we find that

$$
\begin{gathered}
N_{X}=\sum_{k=1}^{N} \int_{Z_{k-1}}^{Z_{k}}\left\{\left(\bar{Q}_{11}\right)_{k}\left(\varepsilon_{x}^{0}+z \kappa_{x}\right)+\left(\bar{Q}_{12}\right)_{k}\left(\varepsilon_{y}^{0}+z \kappa_{y}\right)\right. \\
\left.+\left(\bar{Q}_{16}\right)_{k}\left(\gamma_{x y}^{0}+z \kappa_{x y}\right)\right\} d z
\end{gathered}
$$

and

$$
\begin{gathered}
M_{x}=\sum_{k=1}^{N} \int_{Z_{k-1}}^{Z_{k}}\left\{\left(\bar{Q}_{11}\right)_{k}\left(\varepsilon_{x}^{0}+z \kappa_{x}\right)+\left(\bar{Q}_{12}\right)_{k}\left(\varepsilon_{y}^{0}+z \kappa_{y}\right)\right. \\
\left.+\left(\bar{Q}_{16}\right)_{k}\left(\gamma_{x y}^{0}+z \kappa_{x y}\right)\right\} z d z
\end{gathered}
$$

Combining terms and rearranging Eqs. (10) and (11), we find that

$$
N_{x}=A_{11} \varepsilon_{x}^{0}+A_{12} \varepsilon_{y}^{0}+A_{16} \gamma_{x y}^{0}+B_{11} \kappa_{x}+B_{12} \kappa_{y}+B_{16} \kappa_{x y}
$$

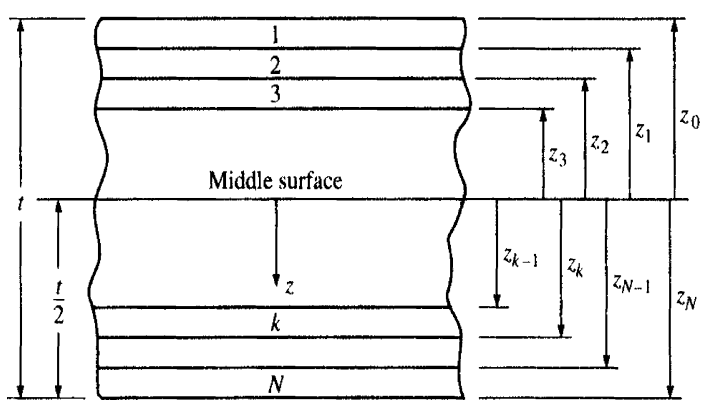

Fig. 5 Laminated plate geometry and ply numbering system

and

$$
M_{x}=B_{11} \varepsilon_{x}^{0}+B_{12} \varepsilon_{y}^{0}+B_{16} \gamma_{x y}^{0}+D_{11} \kappa_{x}+D_{12} \kappa_{y}+D_{16} K_{x y}
$$

where the laminate extensional stiffnesses are given by

$$
A_{i j}=\int_{-t / 2}^{t / 2}\left(\bar{Q}_{i j}\right)_{k} d z=\sum_{k=1}^{N}\left(\bar{Q}_{i j}\right)_{k}\left(Z_{k}-Z_{k-1}\right)
$$

the laminate coupling stiffnesses are given by

$$
B_{i j}=\int_{-t / 2}^{t / 2}\left(\bar{Q}_{i j}\right)_{k} z d z=\frac{1}{2} \sum_{k=1}^{N}\left(\bar{Q}_{i j}\right)_{k}\left(Z_{k}^{2}-Z_{k-1}^{2}\right)
$$

and the laminate bending stiffnesses are given by

$$
D_{i j}=\int_{-t / 2}^{t / 2}\left(\bar{Q}_{i j}\right)_{k} z^{2} d z=\frac{1}{3} \sum_{k=1}^{N}\left(\bar{Q}_{i j}\right)_{k}\left(Z_{k}^{3}-Z_{k-1}^{3}\right)
$$

where the subscript $i, j=1,2$ or 6 . The other stress resultants can be written in a similar form, and the complete set of equations can be expressed in matrix form as

$$
\left\{\begin{array}{l}
N_{x} \\
N_{y} \\
N_{x y} \\
M_{x} \\
M_{y} \\
M_{x y}
\end{array}\right\}=\left[\begin{array}{llllll}
A_{11} & A_{12} & A_{16} & B_{11} & B_{12} & B_{16} \\
A_{12} & A_{22} & A_{26} & B_{12} & B_{22} & B_{26} \\
A_{16} & A_{26} & A_{66} & B_{16} & B_{26} & B_{66} \\
B_{11} & B_{12} & B_{16} & D_{11} & D_{12} & D_{16} \\
B_{12} & B_{22} & B_{26} & D_{12} & D_{22} & D_{26} \\
B_{16} & B_{26} & B_{66} & D_{16} & D_{26} & D_{66}
\end{array}\right]\left\{\begin{array}{l}
\varepsilon_{x}^{0} \\
\varepsilon_{y}^{0} \\
\gamma_{x y}^{0} \\
\kappa_{x} \\
\kappa_{y} \\
\kappa_{x y}
\end{array}\right\}(17)
$$

or in a partitioned form as

$$
\left\{\begin{array}{l}
N \\
M
\end{array}\right\}=\left[\begin{array}{ll}
A & B \\
B & D
\end{array}\right]\left\{\begin{array}{l}
\varepsilon^{0} \\
\kappa
\end{array}\right\}
$$

From Eq. (18), we can see that the extensional stiffness matrix $[A]$ relates the in-plane forces $\{N\}$ to the midplane strain $\left\{\varepsilon^{0}\right\}$ and the bending stiffness matrix $[D]$ relates the moments $\{M\}$ to the curvatures $\{\kappa\}$. The coupling stiffness matrix $[B]$ couples the in-plane forces $\{N\}$ with the curvatures $\{\kappa\}$ and the moments $\{M\}$ with midplane strain $\left\{\varepsilon^{0}\right\}$. A laminate having nonzero $B_{i j}$ will bend or twist under in-plane loads. Such a laminate will also exhibit midplane stretching under bending and twisting moment loading. It can be easily shown that the symmetry of geometric and material properties of the laminate with respect to the middle surface leads to the condition that all $B_{i j}=0$ and that asymmetry about the middle surface leads to nonzero $B_{i j}$. 
Table 1 Material properties of PCBs and components

\begin{tabular}{|c|c|c|c|}
\hline & Pure Cu & FR-4 & EEE Parts \\
\hline $\begin{array}{c}\text { Elastic } \\
\text { Modulus }\end{array}$ & $120 \mathrm{MPa}$ & $22 \mathrm{MPa}$ & - \\
\hline $\begin{array}{c}\text { Tensile } \\
\text { Strength }\end{array}$ & $270 \mathrm{MPa}$ & $30 \mathrm{MPa}$ & - \\
\hline $\begin{array}{c}\text { Mass/ } \\
\text { Density }\end{array}$ & $2.3 \mathrm{~g} / \mathrm{cm}^{\wedge} 3$ & $\begin{array}{c}1.35 \\
\mathrm{~g} / \mathrm{cm}^{\wedge} 3\end{array}$ & 324 grams \\
\hline
\end{tabular}

O'brien has developed an analysis of stiffness reduction in symmetric laminates during delamination based on a simple "rule of mixtures" and the Classical Lamination Theory ${ }^{(12),(13)}$. From Eq. (18) the effective longitudinal Young's modulus of a symmetric laminate is given by

$$
E_{x}=\frac{1}{t A_{11}^{\prime}}
$$

This equation was used by O'brien to model the stiffness of the laminate without delamination. If each layer have the different materials and also totally delaminated laminate, the effective longitudinal Young's modulus is given by ${ }^{(12)}$

$$
E_{t d}=\frac{\sum_{i=1}^{m} E_{x i} t_{i}}{t}
$$

$E_{t d}:$ Equivalent stiffness of PCB

$E_{x i}:$ Nominal stiffness of each layer

$t_{i}$ : Thickness of each layer

$t$ : Total thickness of PCB

According to the above assumption, PCBs mode analysis was performed. For this analysis, MSC/NASTRAN was used as the solver and MSC/PATRAN was used as the pre/post-processor. PCBs were modeled by the isotropic shell elements and electric/electronic parts were modeled as the distributed mass on the PCBs. QFP (Quad Flat Package) having the enough stress relief of lead frame cannot give effect on the stiffness of PCB. But, some electronic parts, for examples, BGA (Ball Grid Array), leadless chip carrier and etc., can deeply give effect on the stiffness of PCB. In our model did not use the BGA, leadless chip carrier and etc., so the distributed mass modeling was available.

To make the exact analytical model for electronic components, mass and elastic modulus has to be reviewed. Firstly, the main materials of PCBs are FR-4 resin and pure copper plates. Also the total mass of electronic components and parts are prepared.

According to the Eq. (20), the calculated copper area density for PCBs nominal size is about $60 \%$. It can transfer to the equivalent thickness having the $100 \%$ area density for the PCBs nominal size as 244.8 micro-meters. Total board thickness is $1.6 \mathrm{~mm}$ for $\mathrm{DM}$ and $2.0 \mathrm{~mm}$ for
PM/FM. If the equivalent thickness of copper layer and FR-4 material substitute to the Eq. (20), then, the equivalent stiffness of PCB is about $30 \mathrm{MPa}$.

The above mass and equivalent stiffness is applied to the structural analysis model.

Figures 6 and 7 show the mode analysis result of DM and PM ( \& FM) board of SAR. Board fundamental frequency was used for the prediction of board deflection under launching force condition. But this study did not show the detailed stress analysis results, and they had enough margin of safety for launching condition.

\section{Review of Random Vibration Test Results}

\subsection{Test configuration}

Random vibration testing became prominent many years ago with the advent of jet engine and rocket motors. Before then, most military standards defined vibration requirements in terms of sinusoidal input forces. For rotary engine applications, this was defined the in-service environment. But spacecraft and launch vehicles rarely see sinusoidal vibration. Rocket engines create wideband acoustic fields, and structural response is random.

To do a random-vibration test, we mount the SAR component on an electro-dynamic shaker, which consists of a large armature whose movement is controlled by an electrical signal evoking a surrounding coil. A test fixture, which attaches to the armature, is necessary to provide a suitable mounting interface for the test article and the relatively constant input at all mounting locations. Typically, an instrument called an accelerometer senses the fixture's and provides real-time feedback for shaker control. But to get the exact response characteristics of PCB for SAR, accelerometer was attached to the PCBs board directly. And we test a component in each of three orthogonal directions.

Figure 8 shows the coordinate system and the position of accelerometers attached to the fixture and SAR unit. Also Fig. 9 shows the $y$-axis test for SAR unit. As shown in Fig. 10, the accelerometer was located at the edge of board.

\section{2 Test results}

To get the detailed response of SAR, seven accelerometers were used. Each of three accelerometers was attached on the test fixture and housing in each perpendicular direction. Normally, board level responses are not needed, but we obtain the response of boards to validate the suggested calculation method for the stiffness of $\mathrm{PCBs}^{(14)}$. The flight article of satellite electronic components does only need the real random vibration test and can be verified with the electrical function test.

Random vibration test was performed per three perpendicular axes, but the results for the normal direction of PCB ( $x$-axis) had the main mode of PCBs and housing. That means, PCB is regarded as the only one dimensional 


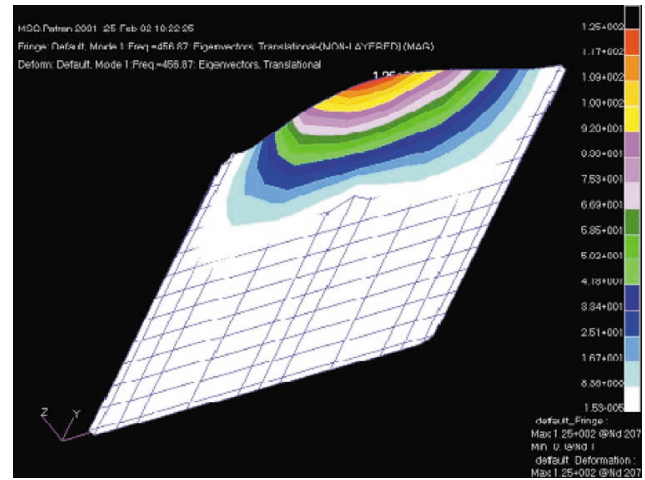

(a) The 1st frequency of SAR 124 board $(456 \mathrm{~Hz})$

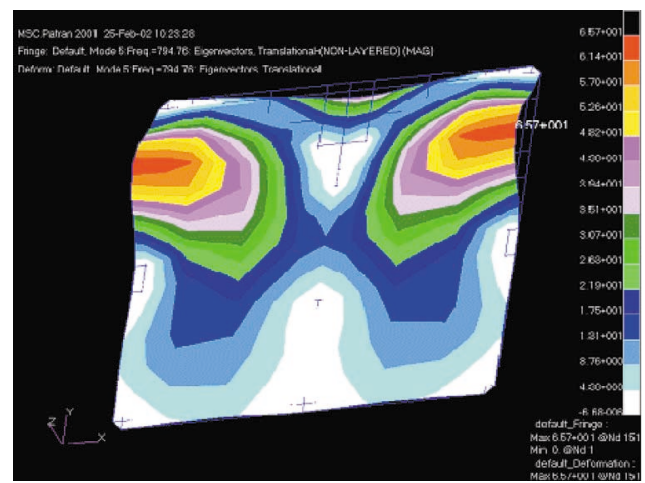

(b) The 3rd frequency of SAR 124 board $(794 \mathrm{~Hz})$

Fig. 6 Mode analysis of SAR PM \& FM

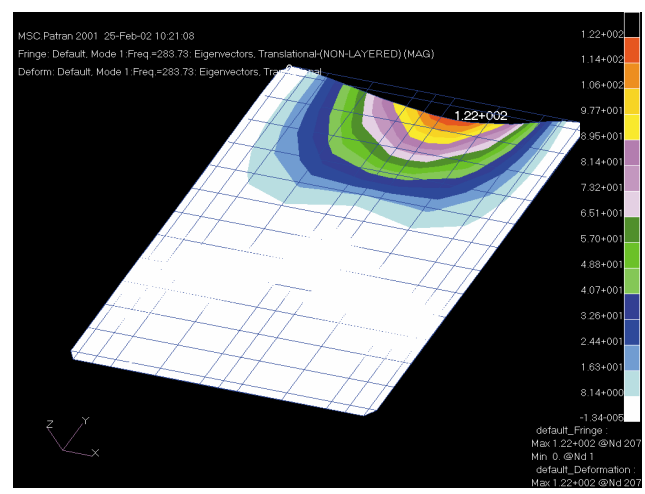

(a) The 1st frequency of SAR 124 board $(283 \mathrm{~Hz})$

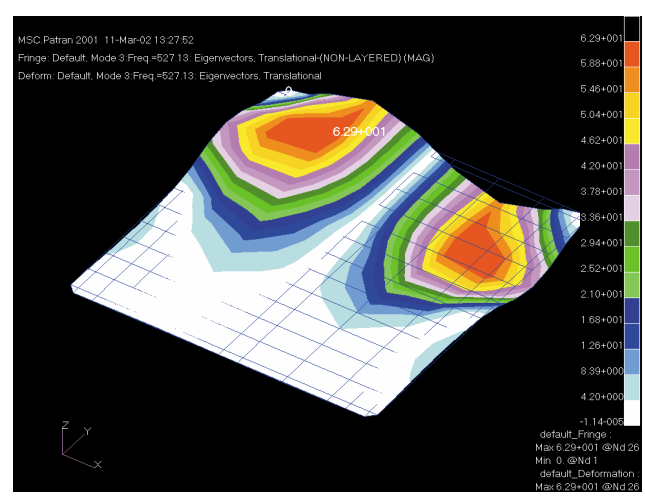

(b) The 3rd frequency of SAR 124 board $(527 \mathrm{~Hz})$

Fig. 7 Mode analysis of SAR DM

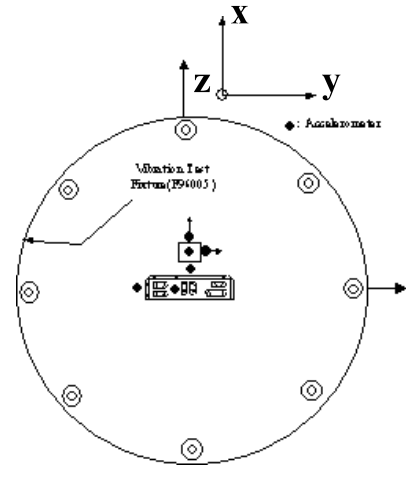

Fig. 8 Vibration test coordinate system

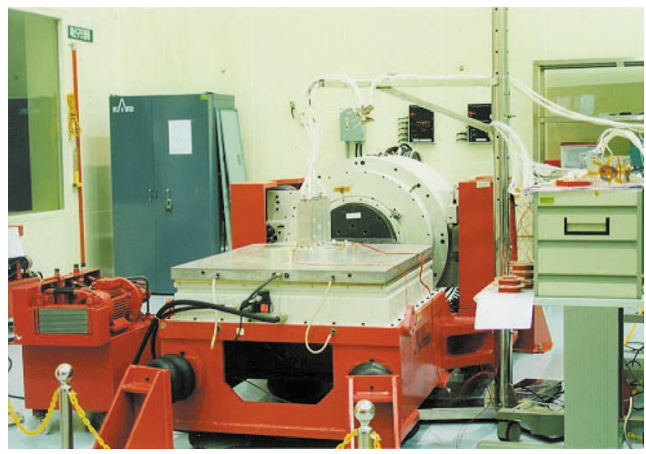

Fig. 9 Configuration of SAR $Y$-Axis random testing

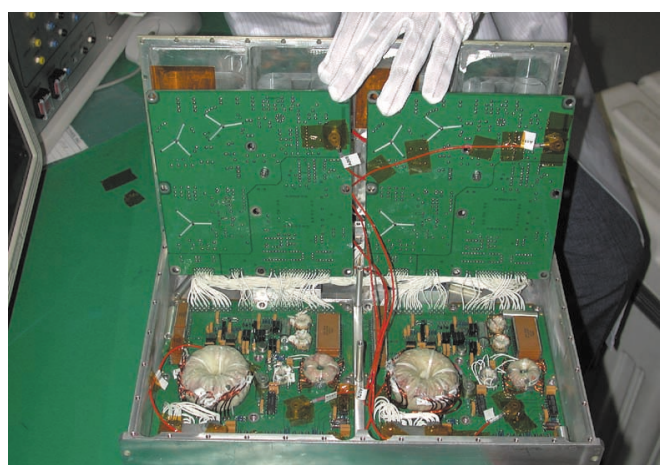

Fig. 10 Accelerometer sensor location on PCB board

vibration system having the only bending response, so the axial direction ( $y \& z$-axis) for PCB is not critical.

There are some limitations to attached to the board with accelerometer. We found the possible area attached the accelerometer, but that location was not occurred the main mode of board. Figure 11 shows the coordinates of PCB board and sensor location attached to the SAR 124 board.

As shown in Fig. 7 (a), the main mode of board was occurred at the edge of board having the large distance between bolts. So the only one accelerometer cannot acquire the response of PCBs main mode. According to the sensor location, the response of 3rd or higher mode of board can be obtained. Figures 6 and 7 (b) show the relative mode for the mounted accelerometer location. 


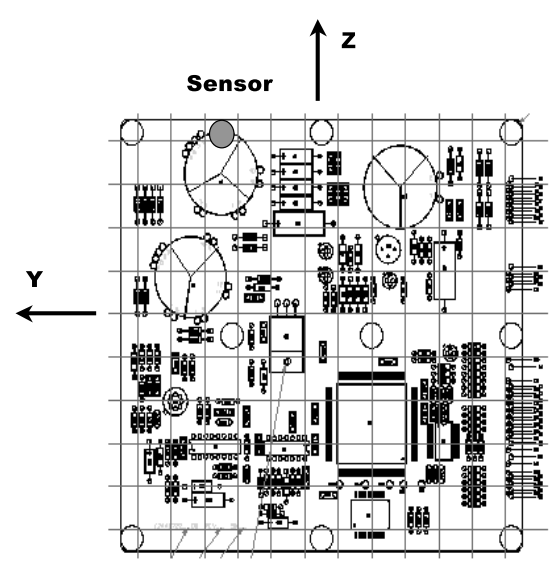

Fig. 11 Sensor location \& coordinate of SAR 124 board

Table 2 Random vibration test input

\begin{tabular}{|c|c|c|c|}
\hline & $\begin{array}{l}\text { Acceptance } \\
\operatorname{PSD}\left(\mathrm{g}^{2} / \mathrm{Hz}\right)\end{array}$ & $\begin{array}{l}\text { Qualification } \\
\operatorname{PSD}\left(\mathrm{g}^{2} / \mathrm{Hz}\right)\end{array}$ & Remarks \\
\hline 20 & 0.013 & 0.026 & \multirow{4}{*}{$\begin{array}{c}\text { 3dB } \\
\text { design } \\
\text { margin }\end{array}$} \\
\hline 50 & 0.08 & 0.16 & \\
\hline 800 & 0.08 & 0.16 & \\
\hline 2000 & 0.013 & 0.026 & \\
\hline
\end{tabular}

Random vibration test profile is referenced by NASA environment specification (GEVS-SE) with $+3 \mathrm{~dB}$ design margin as qualification test level. Table 2 shows the input profile for the random vibration of SAR unit.

Figure 12 shows (a) the input level of test, (b) the result of SAR DM board, and (c) the result of SAR PM boards per normal direction of PCBs.

The above test profile, as shown in Fig. 12 (a), this test profiles can be obtained from NASA specification. Test level is $14.1 \mathrm{Grms}$ for flight article, and duration is two minute per each perpendicular axes ${ }^{(14)}$. As mentioned above chapter, total 7 accelerometers were used for the SAR random vibration test. The following table shows the sensors and location.

Figure 12 (b) shows the response of No.7 sensor only. But Fig. 12 (c) shows the response of No.1 \& 7 sensors. Only one sensor was attached to the PCB as shown in Fig. 11. According to the test results, it can be easily compared with the analysis results as shown in Figs. 6 and 7 . Figure 6 and 7 show the 1st \& 3rd mode of SAR 124 board. But the PCB's response from the sensor attached to the board is deeply related to the 3rd mode of board.

The following table shows the comparison test and analysis results (3rd mode) for KOMPSAT-2 SAR board.

According to the test results, the fundamental frequency of housing is $270 \mathrm{~Hz}$ and $210 \mathrm{~Hz}$ for each $\mathrm{DM}$ $\&$ PM. The following figure shows the fundamental frequency of SAR total unit for DM.

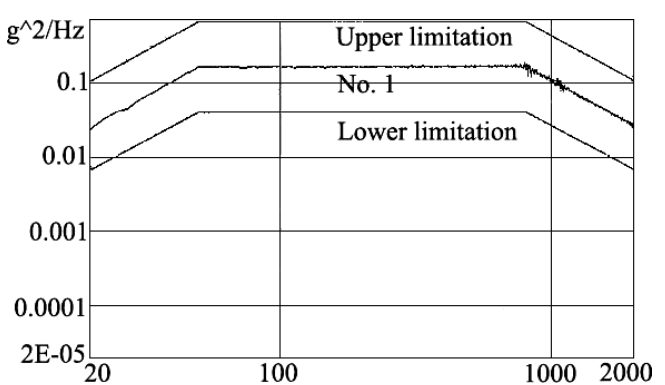

(a) Test input level for $X$ direction

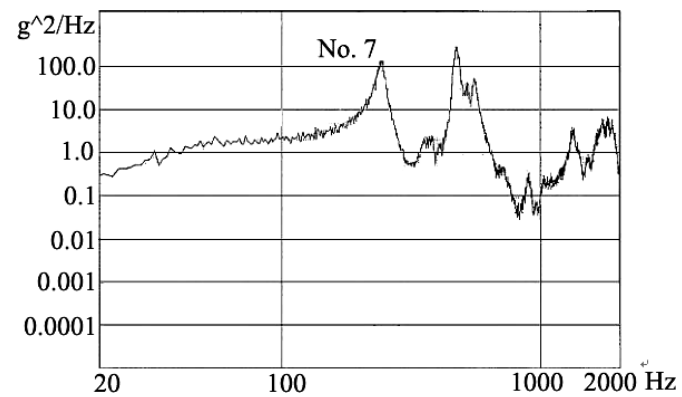

(b) Test result of SAR DM 124 board

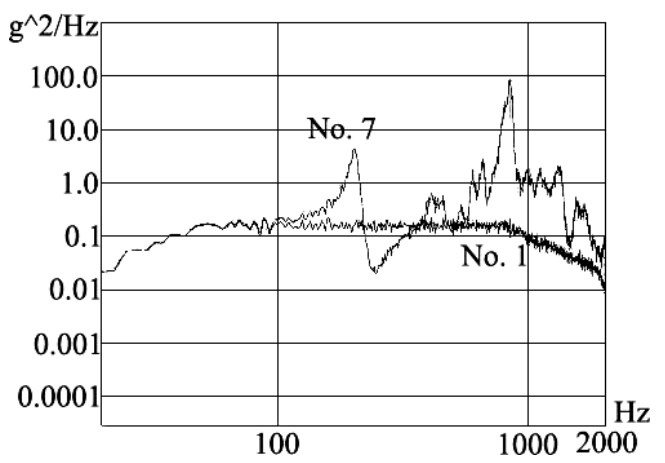

(c) Test result of SAR PM 124 board

Fig. 12 Test results of SAR component

Table 3 Random vibration test input

\begin{tabular}{|c|c|c|}
\hline No. & Sensor Location & Type \\
\hline 1 & $\mathrm{X}$ for fixture & \multirow{7}{*}{$\begin{array}{c}\text { 1-axis } \\
\text { translation } \\
\text { sensor }\end{array}$} \\
\hline 2 & Y for fixture & \\
\hline 3 & $\mathbf{Z}$ for fixture & \\
\hline 4 & $X$ for SAR unit outer surface & \\
\hline 5 & Y for SAR unit outer surface & \\
\hline 6 & $Z$ for $S A R$ unit outer surface & \\
\hline 7 & X for SAR 124 board & \\
\hline
\end{tabular}

\section{Conclusion}

Because of complexity of copper layer, analytical approaches are more complicated and difficult for the multilayered printed circuit board. So we need more reliable method to verify the response of structure of electric board.

In this paper, we suggested that the copper layer pattern is simplified as the volume element using classical composite lamination theory. Using this method, the equivalent stiffness of PCB can be easily obtained. After 
Table 4 Comparison of analysis and test

\begin{tabular}{|c|c|c|c|}
\hline & $\begin{array}{c}\text { ANALYSIS } \\
(\mathrm{Hz})\end{array}$ & $\begin{array}{c}\text { TEST } \\
(\mathrm{Hz})\end{array}$ & $\begin{array}{c}\text { ERROR } \\
(\%)\end{array}$ \\
\hline $\begin{array}{c}\text { SAR DM 124 } \\
\text { board, } \\
\text { 8CU layer }\end{array}$ & 520 & 527 & 1.3 \\
\hline $\begin{array}{c}\text { SAR PM 124 } \\
\text { board, } \\
\text { 10 CU layer }\end{array}$ & 795 & 840 & 5 \\
\hline
\end{tabular}

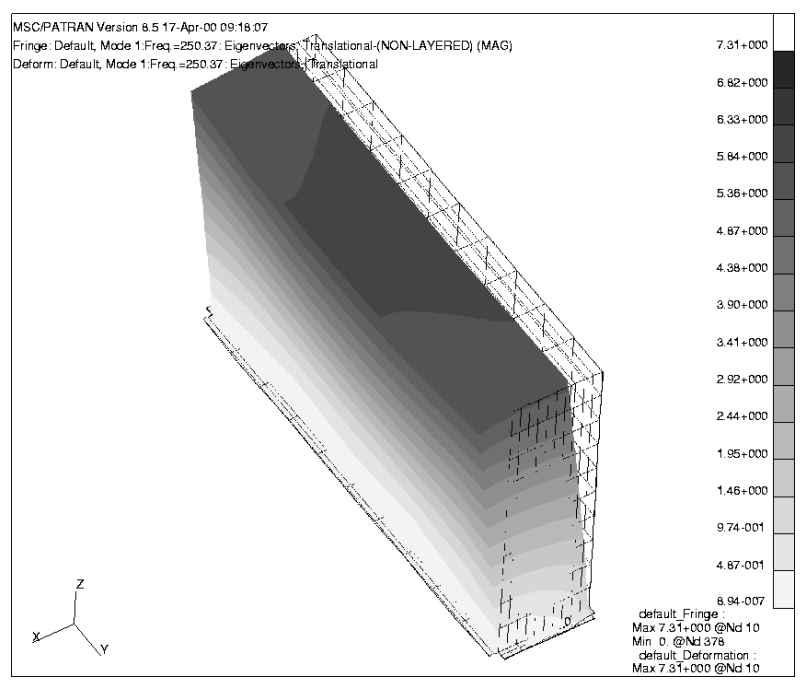

Fig. 13 SAR DM mode analysis result $(250 \mathrm{~Hz})$

the calculation of the equivalent stiffness of board, the data was applied to the analytical model as the 2-dimensional shell elements. First ten modes of PCB were analyzed. And these modes were compared with the test results.

Random vibration testing was supplied for the verification of structural assumption as discussed on the method of PCBs modeling. Vibration test condition was referenced by NASA specification. To get the response of PCBs, accelerometer was mounted on the PCBs surface. Several modes from the accelerometer were acquired. The test result was compared with the analysis results, and the suggestion of the PCB modeling was conservatively verified. The error between the test and analysis results were in 5\% below, so the method was sufficiently verified.

Finally, this study will be a good reference to the similar structural model and a guideline to analyze the compli- cated PCBs using the classical lamination theory for composite materials.

\section{References}

(1) Steinburg, D.S., Vibration Analysis for Electronic Equipment, (1988), pp.220-236, John Wiley \& Sons.

( 2 ) Roberts, J.C. and Stillo, D.M., Random Vibration Analysis of a Printed Wiring Board with Electronic Components, Journal of IES, January/February (1991), pp.25-31.

( 3 ) Barker, D.B., Dasgupta, A. and Pecht, M.G., PWB Solder Joint Life Calculation under Thermal and Vibration Loading, Journal of IES, January/February (1992), pp.17-25.

(4) Sidharth, S. and Barker, D.B., Vibration Induced Fatigue Life Estimation of Corner Leads of Peripheral Leaded Components, Journal of Electronic Packaging, Vol.118 (1996), pp.244-249.

( 5 ) Ham, S.J. and Lee, S.B., Experimental Study for Reliability of Electronic Packaging under Vibration, Experimental Mechanics, Vol.36, No.4 (1996), pp 339-344.

( 6 ) MIL-HDBK-5, Metallic Materials and Elements for Aerospace Vehicle Structures, Department of Defense, Washington, D.C.

( 7 ) Shigley, J.E., Mechanical Engineering Design, (1977), McGraw-Hill.

( 8 ) Blevins, R.D., Formulas for Natural Frequency and Mode Shape, (1979), Krieger Publishing Co.

( 9 ) Tustin, W. and Hieber, G.M., Understanding and Measuring the Shock Response Spectrum, Spectral Dynamics Corporation, Technical Publication SSA-3, October (1975).

(10) Kacena, W.J., McGrath, M.B. and Rader, W.P., "Pyrotechnic Shock Summary", Aerospace Systems Pyrotechnic Shock Data, Volume I, Martin Marietta Corporation for Goddard Space Flight Center, Contract NAS5-15208, March (1970).

(11) Harper, C.A., Electronic Packaging \& Interconnection Handbook, Second Edition, (1997), McGraw-Hill Ltd.

(12) Gibson, R.F., Principles of Composite Material Mechanics, (1994), pp.201-269, McGraw-Hill.

(13) O'brien, T.K., Characterization of Delamination Onset and Growth in a Composite Laminate, Edited by Reifsnider, K.L., Damage in Composite Materials, ASTM STP 775, (1982), pp.140-167, ASTM.

(14) Baumann, R.C., General Environmental Verification Specification for STS \& ELV Payloads, Subsystems, and Components, (1996). 